\title{
Collaborative Control Optimization on Reversible Lanes in Intersection Group under Intelligent Vehicle Infrastructure Cooperative System
}

\author{
Guiliang Zhou $\mathbb{D}^{1},{ }^{1}$ Lina Mao $\mathbb{D}^{1,2}$ Yuke Dai, ${ }^{1}$ Yao Liu, ${ }^{3}$ Xu Bao, ${ }^{1}$ and Pengsen $\mathrm{Hu}^{4}$ \\ ${ }^{1}$ Jiangsu Key Laboratory of Traffic and Transportation Security, Huaiyin Institute of Technology, Huaian 223003, China \\ ${ }^{2}$ School of Transportation, Southeast University, Nanjing 210096, China \\ ${ }^{3}$ Faculty of Management Engineering, Huaiyin Institute of Technology, Huaian 223003, China \\ ${ }^{4}$ Department of Civil and Environmental Engineering, The Pennsylvania State University, State College, PA 16802, USA
}

Correspondence should be addressed to Guiliang Zhou; zglpaper@qq.com and Lina Mao; 30721409@qq.com

Received 24 July 2020; Revised 8 September 2020; Accepted 14 October 2020; Published 5 November 2020

Academic Editor: Zhuping Zhou

Copyright ( 2020 Guiliang Zhou et al. This is an open access article distributed under the Creative Commons Attribution License, which permits unrestricted use, distribution, and reproduction in any medium, provided the original work is properly cited.

Based on the Intelligent Vehicle Infrastructure Cooperative System (IVICS), the paper firstly analyzes the operating characteristics of the intersection group under real-time reversible lanes from the capacity; secondly, the signal phase sequence and signal timing parameters of the intersection are designed, and the green wave control is designed based on the phase difference model. The scheme provides traffic flowing in this section with a continuous green light signal. Finally, taking the intersection of Yongle East Road in Wuxi, Jiangsu Province as an example, it optimizes and controls the green wave of the intersection group and verifies the effectiveness of the phase difference model using the evaluation indicators of the number of stops, green wave speed, traffic capacity, and saturation flow. The results show that the optimization of the number of stops is $9.5 \%$ and the vehicle speed is optimized by $5.3 \%$. The intersection capacity and saturation flow are greatly improved.

\section{Introduction}

Intersections in the urban road network are interacting. In order to achieve efficient and smooth traffic flow, this paper designs a collaborative control optimization method for real-time reversible lane intersection groups under the Intelligent Vehicle Infrastructure Cooperative System (IVICS). Lots of researches have been conducted on reversible lanes at home and abroad. Michael W. Levin and Stephen Levin and Boyles [1] proposed a cell reversal model based on dynamic reversible lanes; based on scheduled intersection control and dynamic variable lanes, they formed a dynamic variable lane control using a single link as a complete program. Assi and Ratrout [2] determined the geometric characteristics that affect the operation of double left-turn lanes. Wael KM Alhajyaseen et al. [3] analyzed the application effect of dynamic lane allocation on intersection paths and established a model combining dynamic lane grouping and the entire intersection signal timing parameters optimization to improve the capacity of the intersection. Assi and Ratrout [2] proposed a method for the rapid allocation of dynamic variable lanes at intersections and this method can use the percentage of turning movement when the traffic enters the entrance of the intersection to predict the best lane group on the spot. Dey et al. [4] discussed the application of reversible lanes on the main road in Washington, DC, and discussed the inherent constraints of the city's external environment and the operational constraints imposed by external stakeholders.

Cheng et al. [5] considered the impact of inbound and outbound traffic on intersection coordination and established a dynamic phase difference model for the intersection. Qu et al. [6, 7], based on the theory of vehicle flow fluctuations, established the intersection phase difference calculation model for the assembly and dissipation of queued vehicles, taking into consideration the interactions 
between intersections and the relationship between traffic demand and supply, and further built a phase difference optimization model from six different conditions of vehicle operation. Cao [8] used traffic overflow and green light empty as the constraints of the phase difference model and adjusted and optimized the phase difference according to whether the green light-on time of two driving directions at the same intersection is synchronized. Jiao [9] used saturation degree as the direction-change switch condition of reversible lanes-if the saturation degree is greater than 0.9 , turn on the direction-change switch-and constructed a dynamic prediction model of intersection traffic flow to predict all directions of car traffic in the next few cycles to determine whether the saturation threshold condition is met. Liu et al. [10] realized the optimization of the intersection signal period and the green signal ratio by establishing the dissipation flow rate model for the interval of the left-turn phase green light signal. Liu et al. [11] constructed a multiobjective optimization model based on the NSGA-II algorithm, using the maximum capacity of intersections and the smallest average delay of vehicles as the objective function to solve the signal timing parameter optimization under reversible lanes. Jiang [12] proposed a method of signal phase sequence combination at each time interval of intersections based on traffic flow indicators. Zeng et al. [13] obtained the scheme of signal timing parameter design at each time interval of intersections in units of time intervals.

At present, the research on the theory of reversible lanes is relatively mature. Scholars have done a lot of research works on the reversible lane change model of intersections, the traffic characteristics of intersections, and the analysis of capacity, but the collaborative control of reversible lanes of intersection groups still needs further research. Specifically, (1) at present, there are few studies on the reversible lanes of intersection groups, and most of the research objects are either single intersection or reversible lanes of main roads, which ignores the connections between intersections; (2) research on the threshold conditions for the change of the attribute of the reversible lanes at the intersection is not enough. Currently, most studies have failed to consider the threshold of the change of the attribute of the reversible lanes under different conditions.

\section{The Characteristics of Effects of Real-Time Reversible Lanes on the Traffic Flow of Intersection Groups}

The setting of real-time reversible lanes greatly improves the crossing capacity of the intersection. In terms of traditional reversible lanes, on the one hand, reversible lanes can increase the capacity of the corresponding phase; on the other hand, if improperly set, it would affect vehicles passing through intersections, reducing the efficiency of vehicles passing through intersections and causing safety problems. The real-time reversible lanes, based on the IVICS, change the direction of traffic flow at intersections, which greatly avoids the waste of road resources.
The capacity is related to the saturation flow and the green signal ratio, and it is generally calculated using the product of the saturation flow and the green signal ratio [14]. Suppose there are $m_{i}$ straight lanes and $n_{i}$ left lanes for the $i$ th intersection of the intersection group. The capacity of the intersection group in the real-time reversible lane setting of the IVICS is as follows.

The capacity of the $i$-th intersection: suppose that, at the $i$-th intersection, there are $x_{i}$ lane attributes changed, $x_{i l}$ represents the number of lanes where the straight direction of the $i$-th intersection changes to the left-turn direction, $x_{i s}$ represents the number of lanes where the left-turn direction of the $i$-th intersection changes to the straight direction, and $x_{i l}, x_{i s}>=0$.

The left-handed phase capacity and saturation flow of the $i$-th intersection are as follows:

$$
\begin{array}{r}
C_{i l}^{\prime}=\left(n_{i}+x_{i l}-x_{i s}\right) \cdot S_{i l}^{\prime} \cdot \frac{g_{i l}^{\prime}}{c_{i}^{\prime}}, \\
S_{i s}^{\prime}=\frac{m_{i}+x_{i s}-x_{i l}}{m_{i}} \cdot S_{i s},
\end{array}
$$

where $C_{i l}^{\prime}$ is the left-hand phase capacity of the $i$-th intersection after setting reversible lanes; $S_{i l}^{\prime}$ is the left-hand phase saturation flow of the $i$-th intersection after setting reversible lanes; $g_{i l}^{\prime}$ is the effective green light duration of the left-hand phase of the $i$-th intersection after setting variable settings; $c_{i}^{\prime}$ is the signal period of the $i$-th intersection after the variable lane is set.

The straight-through phase capacity and saturation flow of the $i$-th intersection are as follows:

$$
\begin{array}{r}
C_{i s}^{\prime}=\left(m_{i}+x_{i s}-x_{i l}\right) \cdot S_{i s}^{\prime} \cdot \frac{g_{i s}^{\prime}}{c_{i}^{\prime}}, \\
S_{i s}^{\prime}=\frac{m_{i}+x_{i s}-x_{i l}}{m_{i}} \cdot S_{i s},
\end{array}
$$

where $C_{i s}^{\prime}$ is the straight-through phase capacity of the $i$-th intersection after the variable lane is set; $S_{i s}^{\prime}$ is the saturation flow of the straight-through phase after the variable lane is set at the $i$-th intersection; $g_{i s}^{\prime}$ is the effective green light duration of the straight phase after the variable lane is set at the $i$-th intersection. Therefore, the capacity of the $i$-th intersection after setting the variable lanes is $C_{i}^{\prime}=C_{i l}^{\prime}+C_{i s}^{\prime}$.

The capacity of the intersection group is

$$
C_{0}^{\prime}=\sum_{i=1}^{k} C_{i}^{\prime} .
$$

\section{Collaborative Optimization of Real-Time Reversible Lanes and Intersection Group Signal}

The traditional signal phase scheme fails to take into account the real-time changes in the traffic flow. The fixed signal phase sequence causes a waste of traffic resources during offpeak hours and traffic congestion during peak hours. The 
general reversible lane signal phase scheme only changes the phase sequence in a fixed time period, which has certain effects on alleviating tidal traffic congestion, but it is difficult to play an effective role in congestion at other times. The real-time reversible lanes of the IVICS consider the real-time running status of the traffic flow and perform real-time collaborative control of the traffic flow at the intersection to improve the efficiency of the traffic flow.

3.1. Signal Phase Sequence Design. The signal phase generally has the following forms [15]: (1) symmetrical release; that is, vehicles in the two driving directions of the intersection synchronously go straight and turn left synchronously, which is the most common signal phase; (2) single-port release; that is, the vehicles in the two driving directions at the intersection are staggered to go straight and turn left. The one direction goes straight, while the other direction stops and waits and then passes after it ends; (3) overlap release; that is, a certain phase is straight, and then the next phase is straight and turn left. These phases are shown in Figures 1-3, respectively.

3.2. Signal Timing Parameter Design. Under the low saturation of the intersection, Webster's best signal cycle model $c_{0}=((1.5 L+5) /(1-Y))$ is mainly used to calculate the signal cycle [7], where $c_{0}$ is the signal cycle, $L$ is the total loss time of the signal cycle, and $Y$ is the sum of the critical phase lane flow ratio; that is, $Y=\sum q_{j} / S_{j}$. After setting the realtime dynamic reversible lanes, the flow ratio between straight and left direction will change, so we need to make some changes to the best signal cycle model, specifically as follows.

The change of the flow ratio of the left-turn phase after the real-time dynamic reversible lane is implemented in the IVICS is

$$
Y_{L}^{\prime}=\frac{q_{l}}{S_{l}^{\prime}}=\frac{q_{l}}{\left(\left(n+x_{l}-x_{s}\right) / n\right) \cdot S_{l}}=\frac{n}{n+x_{l}-x_{s}} \cdot Y_{L}
$$

where $q_{l}$ is the vehicle arrival rate of the left-turn phase of the single intersection, $S_{l}^{\prime}$ represents the saturation flow of leftturn phase of the intersection after the reversible lane is implemented, and $S_{l}$ represents the saturation flow of the left-turn phase of the intersection before the real-time reversible lane is implemented.

After the real-time dynamic reversible lanes are implemented in IVICS, the flow ratio of the straight-through phase becomes

$$
Y_{S}^{\prime}=\frac{q_{s}}{S_{s}^{\prime}}=\frac{q_{s}}{\left(\left(m+x_{s}-x_{l}\right) / m\right) \cdot S_{s}}=\frac{m}{m+x_{s}-x_{l}} \cdot Y_{S},
$$

where $q_{s}$ is the vehicle arrival rate of the left-turn phase of a single intersection, $S_{s}^{\prime}$ represents the saturation flow of the left-turn phase of the intersection after the reversible lane is implemented, and $S_{s}$ represents the saturation flow of the left-turn phase of the intersection before the real-time reversible lane is implemented.
Since the key phase is still straight and left turn after setting the reversible lane, the $L$ value remains. Then, the calculation formula of the optimal signal period of the intersection after setting real-time dynamic reversible lanes under the IVICS is

$c_{0}^{\prime}=\frac{1.5 L+5}{1-\left(Y_{L}^{\prime}+Y_{S}^{\prime}\right)}=\frac{1.5 L+5}{1-\left(Y-\left(n /\left(n+x_{l}-x_{s}\right)\right) \cdot Y_{L}-\left(m /\left(m+x_{s}-x_{l}\right)\right) \cdot Y_{S}\right)}$.

At this time, the key phase of the intersection is the sum of the straight-forward and left-turn phases:

$$
Y^{\prime}=Y-Y_{L}^{\prime}-Y_{S}^{\prime}=Y-\frac{n}{n+x_{l}-x_{s}} \cdot Y_{L}-\frac{m}{m+x_{s}-x_{l}} \cdot Y_{S} \text {. }
$$

After setting the real-time reversible lanes, the effective green light time of the left-turn phase and the straightforward phase is, respectively, as follows: the effective green light time of the left-turn phase $g_{l}^{\prime}=\left(c_{0}^{\prime}-L\right) \cdot Y_{L}^{\prime} / Y^{\prime}$ and the effective green light time of the straight-forward phase $g_{s}^{\prime}=\left(c_{0}^{\prime}-L\right) \cdot Y_{S}^{\prime} / Y^{\prime}$.

Under the high saturation of the intersection, the best signal period calculation formula $c=((1.5 L+5) /(1-0.9))$ is used to calculate the signal period. The calculation formula of the effective green light duration is similar to that of the low saturation.

3.3. Collaborative Optimization of Space-Time Resources of Intersection Group. The purpose of establishing the collaborative optimization model of the space-time resources of the intersection group under the IVICS is to improve the intersection group's passing capacity. The key to improving the intersection group's passing capacity lies in the critical path; by giving the "green wave" signal to the heavy traffic flow direction, vehicles in the direction of heavy traffic can get a continuous green light signal and pass through all intersections on the road without hindrance. The traditional green wave cooperative control method is mainly a graphical method. This method is difficult to obtain a two-way green wave scheme, the resulting green wave bandwidth is relatively narrow, and the green wave speed is relatively low, which is difficult to meet the designed speed required by the main traffic line. In this paper, the green wave control is realized by establishing the calculation model of the phase difference between the intersections on the mainline.

$\mathrm{Qu}$ et al. [6] analyzed the theory of vehicle flow fluctuations from the perspective of kinematics and obtained the wave velocity equations for the propagation of parking and starting waves. The wave velocity formulas are, respectively,

$$
\begin{aligned}
& v_{b}=-\frac{v_{0}}{h_{0} \cdot k_{j} \cdot v_{0}-1}, \\
& v_{d}=-\frac{v_{1}}{h_{1} \cdot k_{j} \cdot v_{1}-1} .
\end{aligned}
$$

Here, $v_{b}$ is the speed of the parking wave, $v_{d}$ is the starting wave speed, $v_{0}$ is the initial driving speed of the vehicle, $v_{1}$ is the driving speed of the vehicle after the queue 


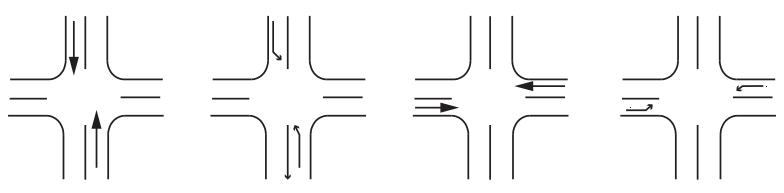

Figure 1: Symmetrical release phase.

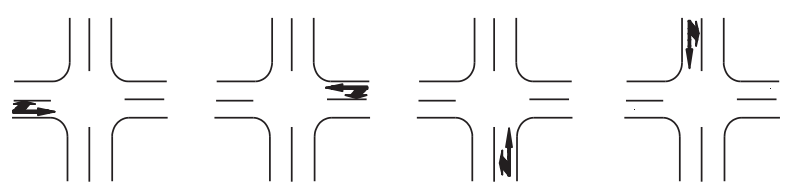

FIgURE 2: Single-port release phase.

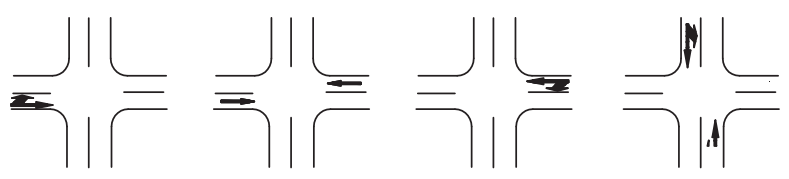

FIGURE 3: Overlap release phase.

is dissipated, $k_{j}$ is the blocking density (the density of the traffic that is too dense to drive normally), $h_{0}$ is the initial headway, and $h_{1}$ is the headway after vehicle queue is dissipated. It can be seen from the trajectory diagram of the traffic flow that the position of the longest queue length is the position where the parking wave meets the starting wave.

3.3.1. Phase Difference Calculation. Let $L_{i-1 . i}$ be the distance between the $i$-1th intersection and the $i$-th intersection in the intersection group and $v_{i-1, i}$ be the travel speed between the i- 1 th intersection and the $i$-th intersection in the intersection group; then the time required for the vehicle to pass through these two intersections is $t_{i-1, i}=L_{i-1, i} / v_{i-1, i}$. The number of vehicles in the queue can be calculated:

$$
N_{i-1, i}=\sum_{j=1}^{2} K_{i, j} \cdot L_{i, j},
$$

where $K_{i, j}$ is the traffic density of the $j$-th phase of the $i$-th intersection and $L_{i, j}$ is the length of the queue ( $j=1$, representing the straight phase; $j=2$, representing the left-turn phase). The queuing time of traffic flow at the intersection is the sum of the queuing formation time and the queuing dissipation time. The queuing vehicle dissipation time is $t_{i-1, i}=N_{i-1, i} / C$, where $C$ is the capacity of the intersection, and the queuing formation time is the time period when the first vehicle starts queuing and sends the parking wave from the stopping line backward to the following vehicles until it restarts and sends the starting wave backward. The phase difference between the intersection $i-1$ and the intersection $i$ is

$$
\phi_{i-1, i}=\frac{L_{i-1, i}}{v_{i-1, i}}-\frac{N_{i-1, i}}{C} .
$$

3.3.2. Optimization of Phase Difference Model. The optimization of the phase difference model in the IVICS is mainly to enable vehicles with different speeds to pass through all intersections along the way without queuing and parking. If the vehicle speed is lower than the target speed, the intelligent vehicular facility guides to increase its speed to be the speed to pass the intersection. If the vehicle speed is higher than the target speed, then it guides to reduce the vehicle speed to be the speed to pass the intersection without queuing. According to the definition of phase difference [16], the phase difference is the time difference between the red and green lights on the upstream and downstream intersections. Therefore, the optimization of the phase difference is mainly from the time of the green or red lights between the upstream and downstream intersections. According to the three methods of green wave cooperative control, there are three main ways to turn on the signal lights between intersections: the signal lights between the upstream and downstream intersections are on at the same time, opposite, and mixed. The phase difference optimization formulas in the three cases are derived similarly, so the 
phase difference optimization is mainly discussed in the case that the signal of the intersection is synchronized.

When the signal lights of the upstream and downstream intersections are synchronized and the signal lights of the upstream and downstream intersections are simultaneously red, the traffic flow of the downstream intersections starts forming a queue from the parking line and forms a parking wave to pass to the upstream intersection. Meanwhile, the upstream intersection turns into a red light, causing the traffic to cut off and the vehicle cannot pass through. Therefore, when the parking wave reaches the end of the queue, the queue length is the longest and remains for a period of time. Its vehicle trajectory is shown in Figure 4 . Let $L_{D}^{i-1, i}$ be the distance from the detector to the downstream intersection; let $L_{\max }^{i-1, i}$ be the maximum queuing length; $t_{A}^{i-1, i}$ is the time when the parking wave transmits to the detector; $t_{B}^{i-1, i}$ is the time when the starting wave is transmitted to the detector; $t_{C}^{i-1, i}$ is the time when the vehicle at the end of the queue travels to the detector when the vehicle flow dissipates; $L_{D E}^{i-1, i}$ is the distance from the detector to the tail of the traffic queue; $T_{\max }^{i-1, i}$ is the moment when the maximum queuing length is formed; $v_{b}^{i-1, i}$ is the speed of the parking wave due to parking when the vehicle reaches the parking line; $v_{d}^{i-1, i}$ is the speed of the starting wave when the queuing vehicle starts moving from the parking line; $v_{q}^{i-1, i}$ is the speed of the vehicle when it is queued to dissipate; $v_{d}^{i-1, i}$ is the speed of the starting wave of the next cycle; $T_{R}^{i-1, i, n}$ is the moment when the red light turns on in the $n$-th cycle; $T_{G}^{i-1, i, n}$ is the moment when the green light turns on in the $n$-th cycle; $T_{R}^{i-1, i, n+1}$ is the moment when the red light of the $n+1$-th cycle turns on.

Suppose that the moment when the vehicle queued at the tail of the traffic flow moves to the parking line of the downstream intersection is $T_{T}^{i-1, i}$; then two situations may occur when the vehicle at the tail of the queue reaches the parking line. The first is $T_{T}^{i-1, i}<=T_{R}^{i-1, i, n+1}$; that is, the vehicle can pass through the parking line before the red light turns on at the next cycle, and then all the queued vehicles can pass through the intersection. The second case is $T_{T}^{i-1, i}>T_{R}^{i-1, i, n+1}$. If the queued vehicles cannot fully pass through the intersection, a secondary queue or stranded queue will be formed, and the queue length is $L_{\min }^{i-1, i}$.

It can be seen from the queuing time interval that

$$
L_{\max }^{i-1, i}=L_{D}^{i-1, i}+L_{D E}^{i-1, i}
$$

and

$$
\left\{\begin{aligned}
v_{d}^{i-1, i}= & \frac{L_{D E}^{i-1, i}}{T_{\max }^{i-1, i}-t_{B}^{i-1, i}}, \\
v_{q}^{i-1, i}= & \frac{L_{D E}^{i-1, i}}{t_{C}^{i-1, i}-T_{\max }^{i-1, i}}, \\
& \frac{v_{d}^{i-1, i} \cdot v_{q}^{i-1, i}}{v_{d}^{i-1, i}+\frac{i-1, i}{q}}
\end{aligned}\right.
$$

thus calculating

$$
L_{D E}^{i-1, i}=\frac{v_{d}^{i-1, i} \cdot v_{q}^{i-1, i}}{v_{d}^{i-1, i}+v_{q}^{i-1, i}}\left(t_{C}^{i-1, i}-t_{B}^{i-1, i}\right)
$$

and getting

$$
L_{\max }^{i-1, i}=L_{D}^{i-1 . i}+\frac{v_{d}^{i-1, i} \cdot v_{q}^{i-1, i}}{v_{d}^{i-1, i}+v_{q}^{i-1, i}}\left(t_{C}^{i-1, i}-t_{B}^{i-1, i}\right) .
$$

In addition, the moment when the vehicle at the end of the queue of the traffic flow passes the parking line of the lane is

$$
\begin{array}{r}
T_{T}^{i-1, i}=T_{\max }^{i-1, i}+\frac{L_{\max }^{i-1, i}}{v_{q}^{i-1, i}}, \\
v_{d}^{i-1,1^{\prime}}=\frac{L_{\min }^{i-1, i}}{T_{\min }^{i-1, i}-T_{R}^{i-1, i, n+1}}, \\
v_{q}^{i-1, i}=\frac{L_{\min }^{i-1, i}}{T_{T}^{i-1, i}-T_{\min }^{i-1, i}}, \\
\frac{v_{d}^{i-1,1^{\prime}} \cdot v_{q}^{i-1, i}}{v_{d}^{i-1,1^{\prime}}+v_{q}^{i-1, i}} .
\end{array}
$$
is

The length of the stranded queue or the secondary queue is

$$
L_{\min }^{i-1, i}=\frac{v_{d}^{i-1,1^{\prime}} \cdot v_{q}^{i-1, i}}{v_{d}^{i-1,1^{\prime}}+v_{q}^{i-1, i}}\left(T_{T}^{i-1, i}-T_{R}^{i-1, i, n+1}\right)=\frac{v_{d}^{i-1,1^{\prime}} \cdot v_{q}^{i-1, i}}{v_{d}^{i-1,1^{\prime}}+v_{q}^{i-1, i}}\left(T_{\max }^{i-1, i}+\frac{L_{\max }^{i-1, i}}{v_{q}^{i-1, i}}-T_{R}^{i-1, i, n+1}\right) .
$$

In order to prevent the traffic overflow phenomenon of queued traffic, the signal lights of the downstream intersection need to be kept for a period of time for the vehicles in the queue to dissipate. Therefore, there may be two extreme cases. The first is that the traffic from the upstream intersection crosses the intersection when the traffic light at the intersection turns green; no queuing happens when driving to the end of the queue at the downstream intersection. In this case, when the upstream traffic flows to the end of the queue, the parking wave and the starting wave at the downstream intersection meet; the phase difference needs to be met at this time: the traffic at the upstream intersection does not line up when passing through the downstream intersection; that is, 


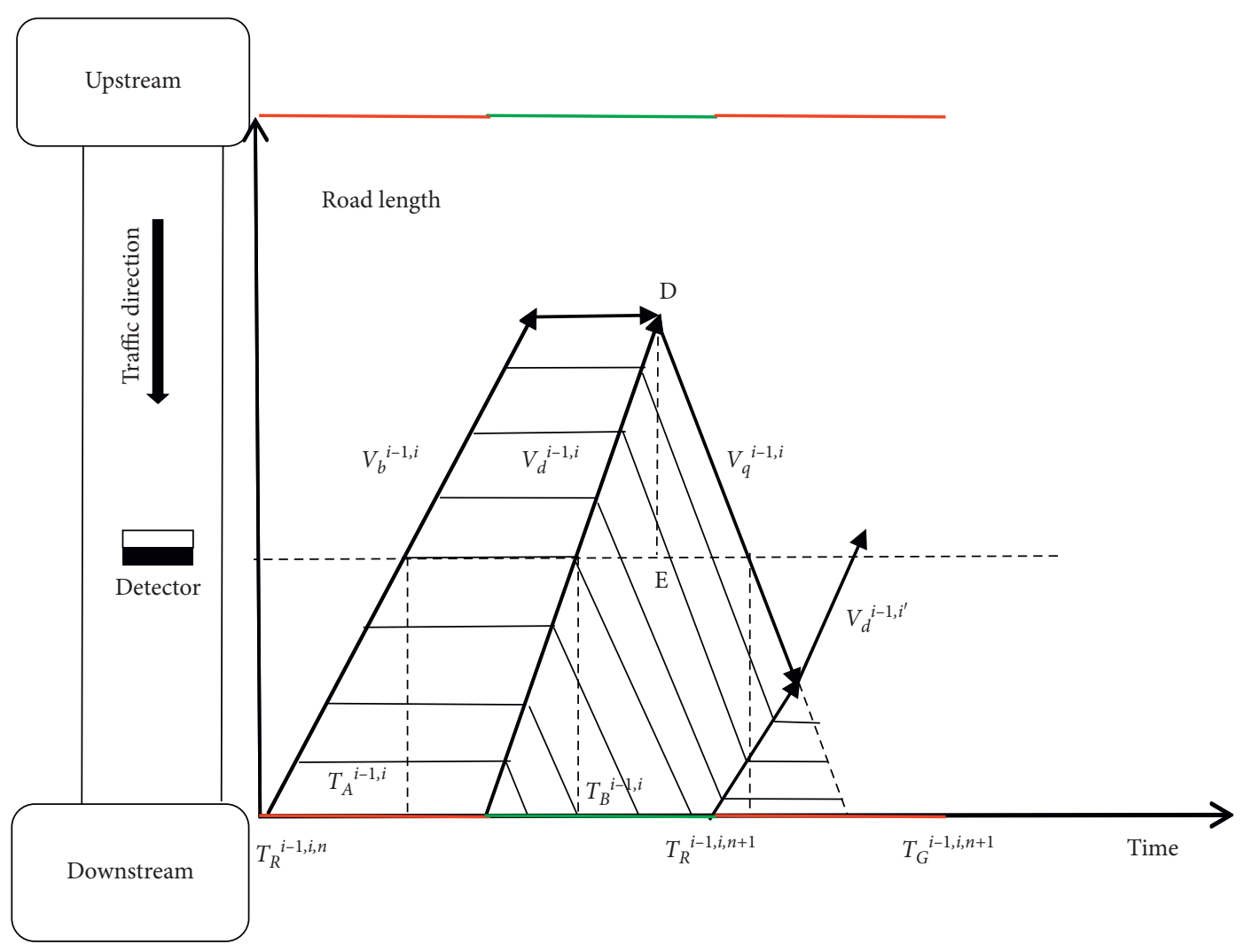

FIGURE 4: Vehicle trajectory when the intersection signals are synchronized.

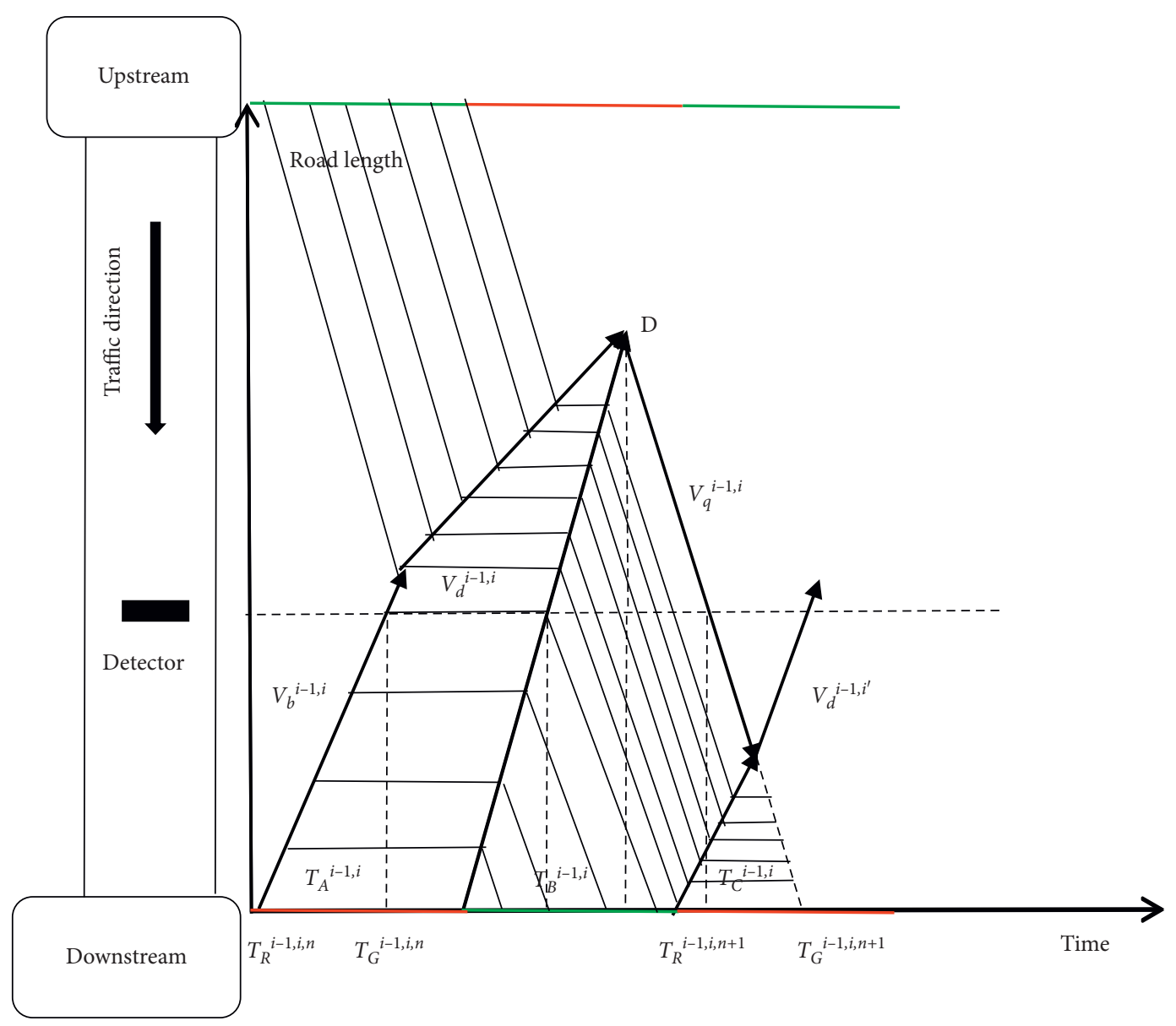

FIGURE 5: Vehicle trajectory when the intersection signals are controlled oppositely. 


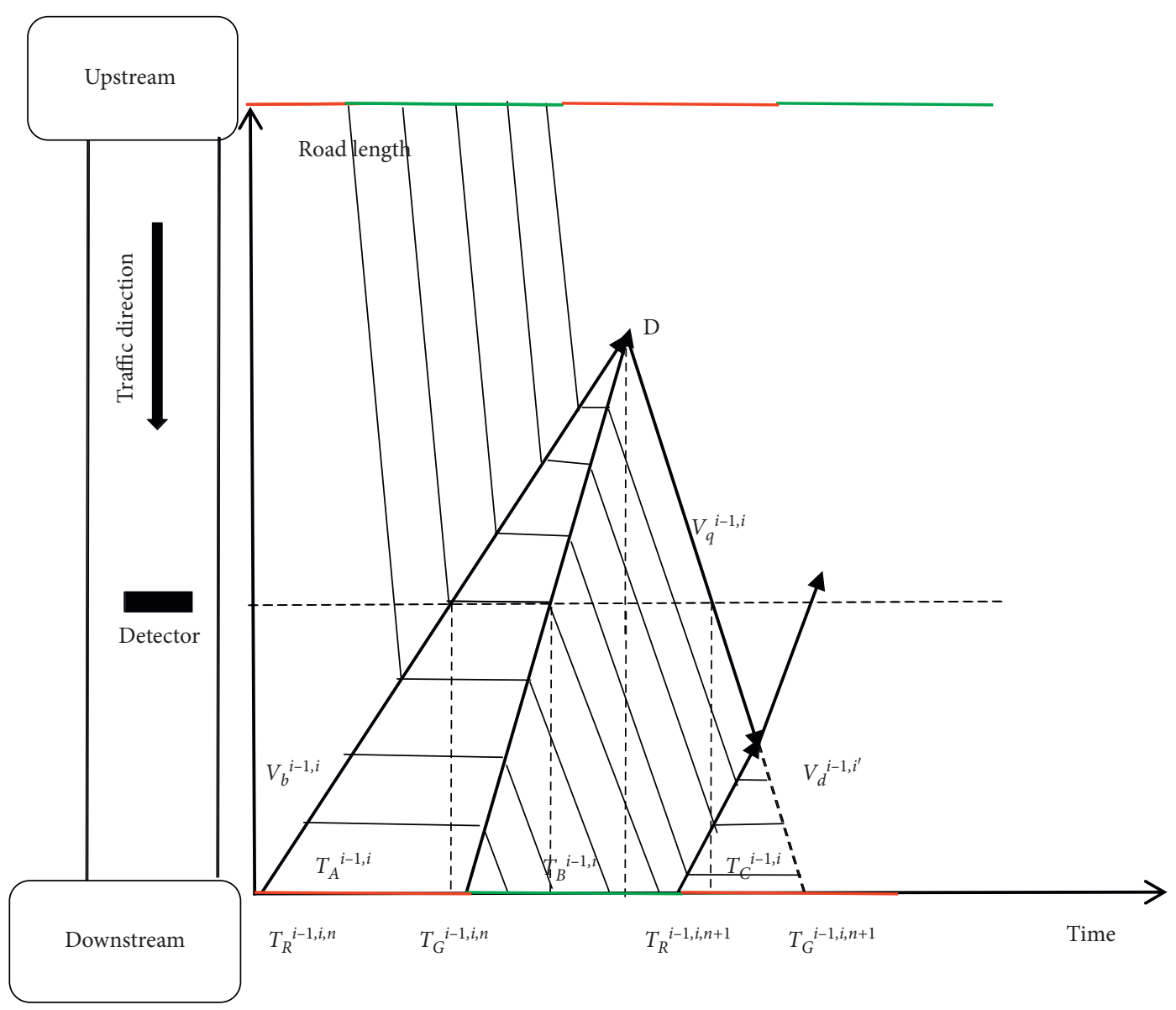

Figure 6: Vehicle trajectory when the intersection signals are mixed and controlled.

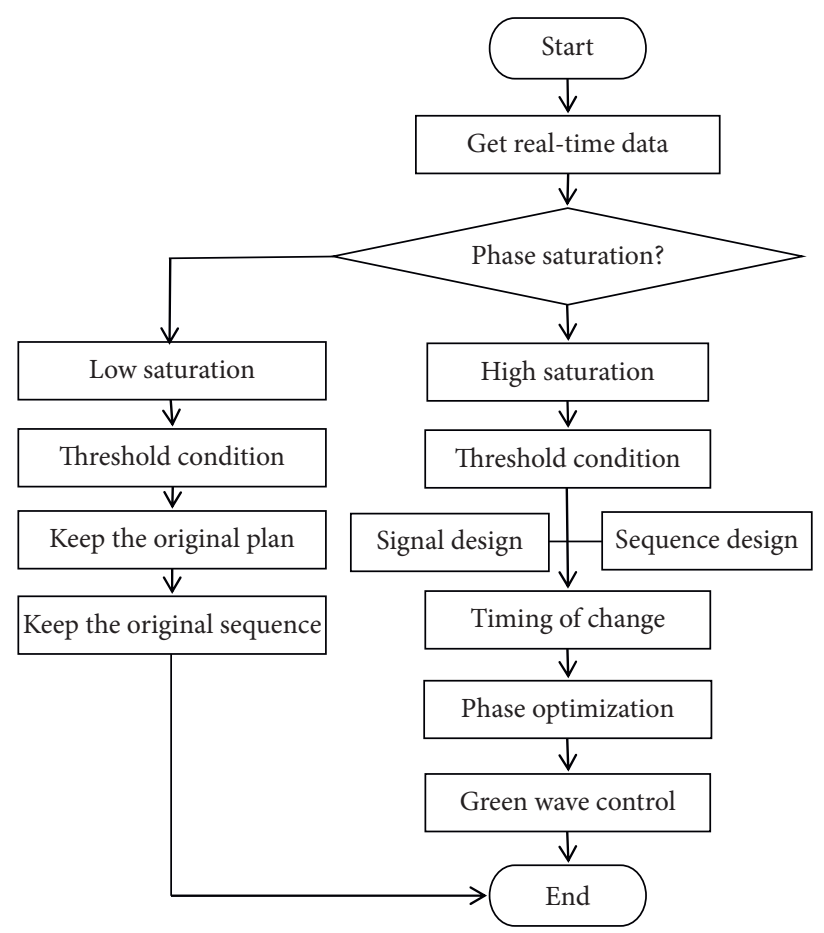

FIgURE 7: Chart of dynamic control. 


$$
\phi_{i-1, i}+\frac{L_{\max }^{i-1, i}}{v_{d}^{i-1, i}}=\frac{L_{i-1, i}+L_{i-1}-L_{\max }^{i-1, i}}{v_{i-1, i}} .
$$

The second case is that when the traffic at the upstream intersection reaches the parking line at the downstream intersection, the vehicles at the end of the queue at the downstream intersection just pass through the parking line. At this time, the phase difference needs to be satisfied: the traffic at the upstream intersection just crosses the parking line at the downstream intersection; that is,

$$
\phi_{i-1, i}+\frac{L_{\min }^{i-1, i}}{v_{d}^{i-1, i}}+\frac{L_{\min }^{i-1, i}}{v_{q}^{i-1, i}}=\frac{L_{i-1, i}+L_{i-1}}{v_{i-1, i}} .
$$

We can know

$$
\phi_{i-1, i}=\frac{L_{i-1, i}+L_{i-1}}{v_{i-1, i}}-\frac{L_{\min }^{i-1, i}}{v_{d}^{i-1, i}}-\frac{L_{\min }^{i-1, i}}{v_{q}^{i-1, i}} .
$$

Therefore, when, under the IVICS, the traffic flow at the upstream intersection by getting a green light signal can pass through the downstream intersection without stopping, the phase difference needs to meet

$$
\frac{L_{i-1, i}+L_{i-1}-L_{\max }^{i-1, i}}{v_{i-1, i}}-\frac{L_{\max }^{i-1, i}}{v_{d}^{i-1, i}}<=\phi_{i-1, i}<=\frac{L_{i-1, i}+L_{i-1}}{v_{i-1, i}}-\frac{L_{\min }^{i-1, i}}{v_{d}^{i-1, i}}-\frac{L_{\min }^{i-1, i}}{v_{q}^{i-1, i}}
$$

It can be seen from the three methods of green wave cooperative control that there are three main ways to turn on the signal lights between intersections. The derivation of the optimization formula of the phase difference is similar under the three situations of the same, opposite, and mixed interaction of the signal lights between the upstream and downstream intersections. The derivation of the phase difference optimization formula of the opposite and mixed interaction is omitted here. The vehicle trajectory when the intersection signal control is opposite is shown in Figure 5, and the vehicle trajectory when the intersection signal control is mixed is shown in Figure 6.

3.4. Real-Time Dynamic Control Flow of Reversible Lanes Intersection Groups. The attribute change of the real-time reversible lanes is determined by detecting the traffic volume on each phase entrance lane by the detectors set at each intersection. If the traffic at the intersection phase is not saturated, it means that the left-turn phase or the straightforward phase still has another phase of the available lane that is more congested; if the traffic at a certain phase of the intersection has reached saturation, then the intersection signal timing parameter design needs to be optimized. The specific process is as follows (see Figure 7):

(1) Obtain real-time traffic flow and queuing length data through the detector set at real-time reversible lane intersections.

(2) The information control center analyzes and processes these initial data to obtain key data such as flow ratio.
(3) Determine the phase saturation of the real-time reversible lane intersection and determine whether the intersection status is high or low saturation.

(4) Substitute the threshold condition to see if the condition for turning on the change switch of the real-time reversible lane is satisfied. If it is satisfied, the number of lanes and the time for turning on the real-time reversible lane change are determined; otherwise, the original signal timing scheme is maintained.

(5) The phase difference is optimized to realize green wave control.

\section{Case Study}

Taking Yongan East Road in Wuxi City, Jiangsu Province, as the research object, the selected intersections are the intersections of Tongyang Road and Yongle East Road, Tangnan Road and Yongle East Road, and Xingyuan Middle Road and Yongle East Road. Yongle East Road (Tongyang Road to Xingyuan Middle Road Section) is a two-way sixlane road. The signal phase sequence and signal timing parameters of the intersection are shown in Table 1. The capacity and saturation flow are shown in Tables 2 and 3. The intersection of Yongle East Road is now under collaborative control, and green wave control is designed to improve the operating efficiency of the Yongle East Road intersection. The optimized signal phase sequence and signal timing parameters are shown in Table 4.

Take the intersection of Tongyang Road and Yongle East Road as the reference intersection, and then calculate the phase difference of the intersection of Tangnan Road and Yongle East Road and the intersection of Xingyuan Middle Road and Yongle East Road with respect to the reference intersection to form green wave traffic. The phase difference between the intersections of Tongyang Road and Yongle East Road set as a reference intersection is zero; the phase difference of the intersection of Tangnan Road and Yongle East Road relative to the intersection of Tongyang Road and Yongle East Road is 38.2 seconds. The phase difference of the intersection of Xingyuan Middle Road and Yongle East with respect to the intersection of Tongyang Road and Yongle East Road is 52.8 seconds. The green wave control chart is shown in Figure 8. It can be seen from Figure 8 that the green wave bandwidth is $36 \mathrm{~s}$, and it can be calculated that the average green wave speed is $37.9 \mathrm{~km} / \mathrm{h}$.

The evaluation indicators for the effect of the implemented green wave control are the number of stops, green wave bandwidth, green wave vehicle speed, capacity, and saturation flow. The effectiveness of the green wave control is verified by calculating and comparing the evaluation indexes of Yongle East Road before and after implementing the green wave control. See Table 5-7 for the comparison of the relevant indicators before and after optimization.

It can be seen from Table 5 that, after the signal timing parameter optimization of the intersection on Yongle East Road, the number of stops, at the intersection, has been reduced to a certain extent, with an average reduction of 
TABle 1: Signal phase sequence before optimization.

Intersection parameter

TAble 2: Capacity.

\begin{tabular}{|c|c|c|c|}
\hline Intersection parameter & Turn left & Straight & Turn right \\
\hline Intersection of Tongyang Road and Yongle East Road & 124 & 726 & 363 \\
\hline Intersection of Tangnan Road and Yongle East Road & 348 & 830 & 415 \\
\hline Intersection of Xingyuan Middle Road and Yongle East Road & 166 & - & 526 \\
\hline
\end{tabular}

TABle 3: Saturation flow.

\begin{tabular}{|c|c|c|c|}
\hline Intersection parameter & Turn left & Straight & Turn right \\
\hline Intersection of Tongyang Road and Yongle East Road & 279 & 653 & - \\
\hline Intersection of Tangnan Road and Yongle East Road & 822 & 653 & - \\
\hline Intersection of Xingyuan Middle Road and Yongle East Road & 415 & - & 658 \\
\hline
\end{tabular}

TABle 4: Optimized signal phase sequence.

Intersection parameter

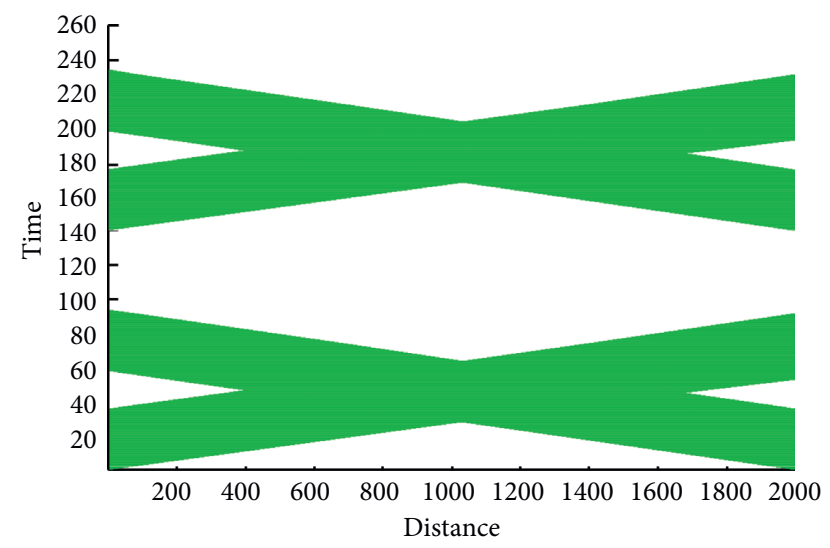

Figure 8: Green wave time interval. 
TABLE 5: Number of stops before and after optimization.

\begin{tabular}{lcc}
\hline Intersection status & Before optimization & Optimized \\
\hline Intersection of Tongyang Road and Yongle East Road & 163 & 124 \\
Intersection of Tangnan Road and Yongle East Road & 118 & 79 \\
Intersection of Xingyuan Middle Road and Yongle East Road & 56 & 102 \\
\hline
\end{tabular}

TABle 6: Optimized capacity at intersections.

\begin{tabular}{|c|c|c|c|}
\hline Intersection parameter & Turn left & Straight & Turn right \\
\hline Intersection of Tongyang Road and Yongle East Road & 132 & 770 & 385 \\
\hline Intersection of Tangnan Road and Yongle East Road & 350 & 832 & 416 \\
\hline Intersection of Xingyuan Middle Road and Yongle East Road & 169 & - & 536 \\
\hline
\end{tabular}

TABLE 7: Comparison of entrance capacity before and after optimization.

\begin{tabular}{lcc}
\hline Intersection parameter & Before optimization & Optimized \\
\hline Intersection of Tongyang Road and Yongle East Road & 850 & 902 \\
Intersection of Tangnan Road and Yongle East Road & 1011 & 1182 \\
Intersection of Xingyuan Middle Road and Yongle East Road & 692 & 705 \\
\hline
\end{tabular}

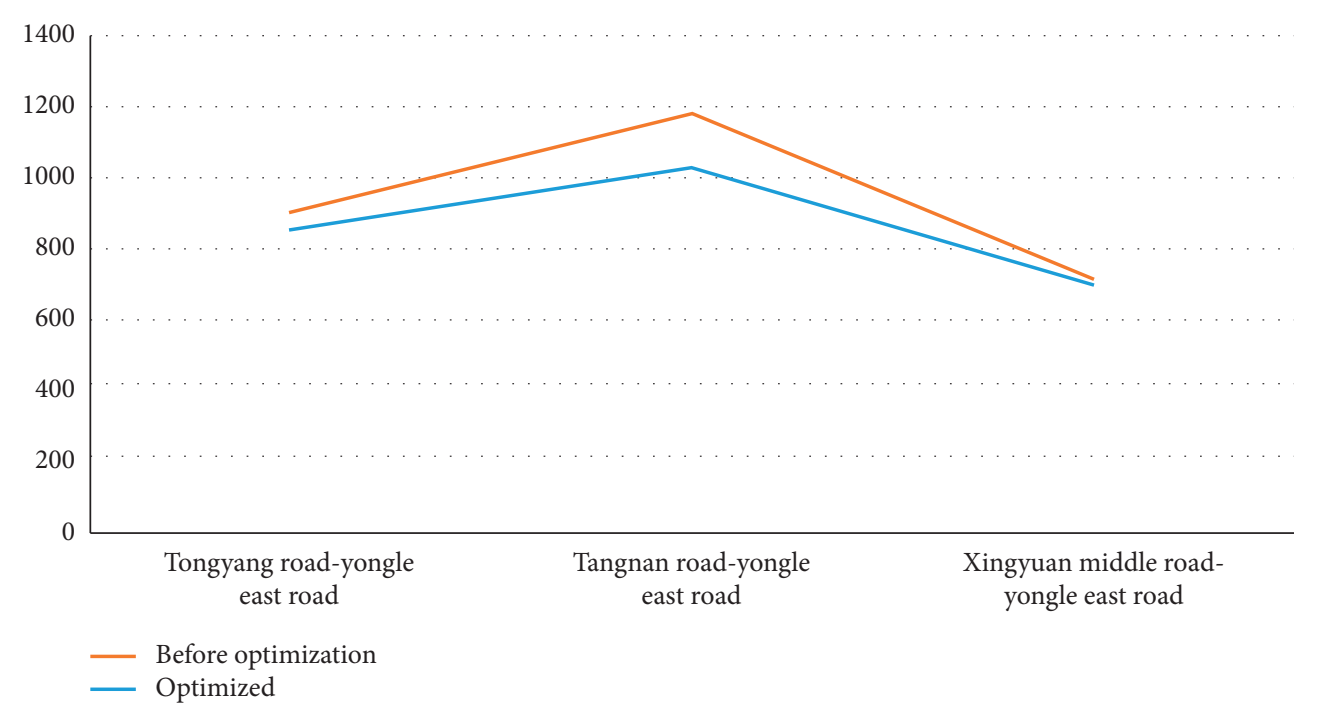

Figure 9: Comparison chart of capacity.

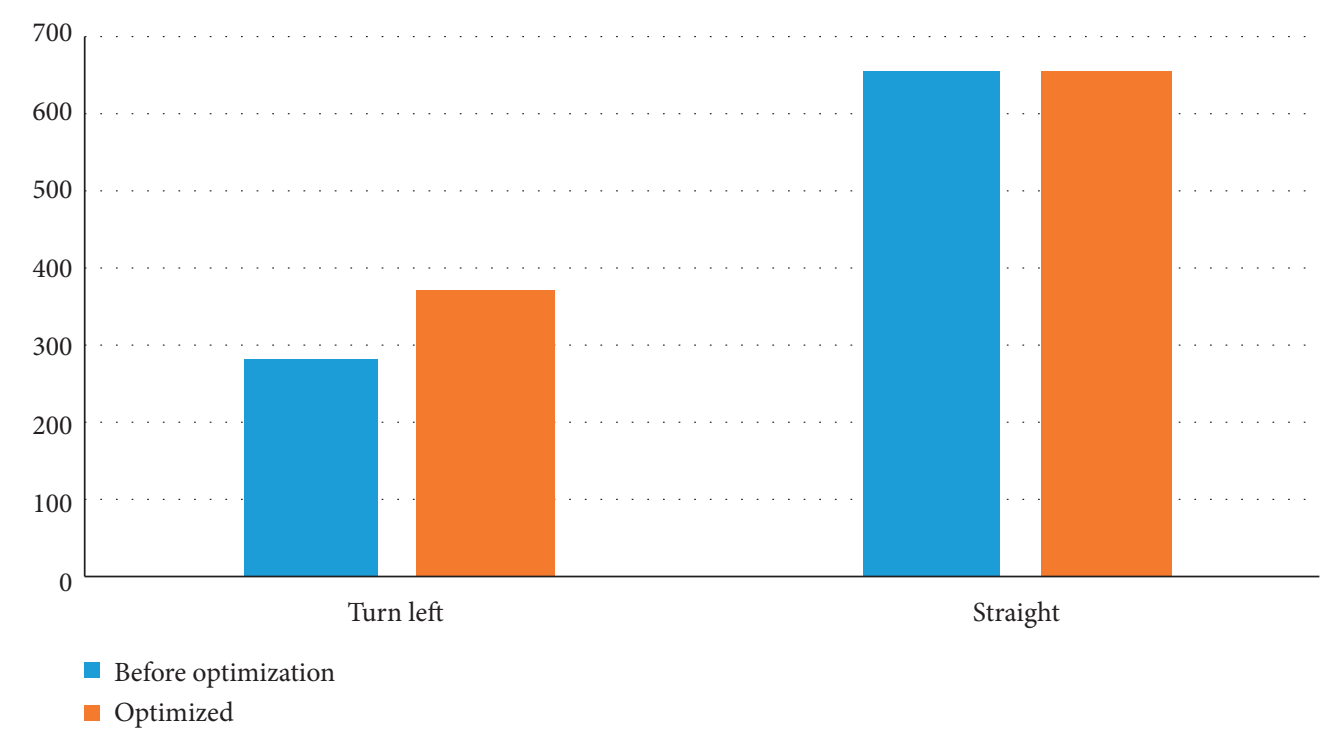

FIgURE 10: Comparison of saturation flow of each phase of Tongyang Road and Yongle East Road before and after optimization. 


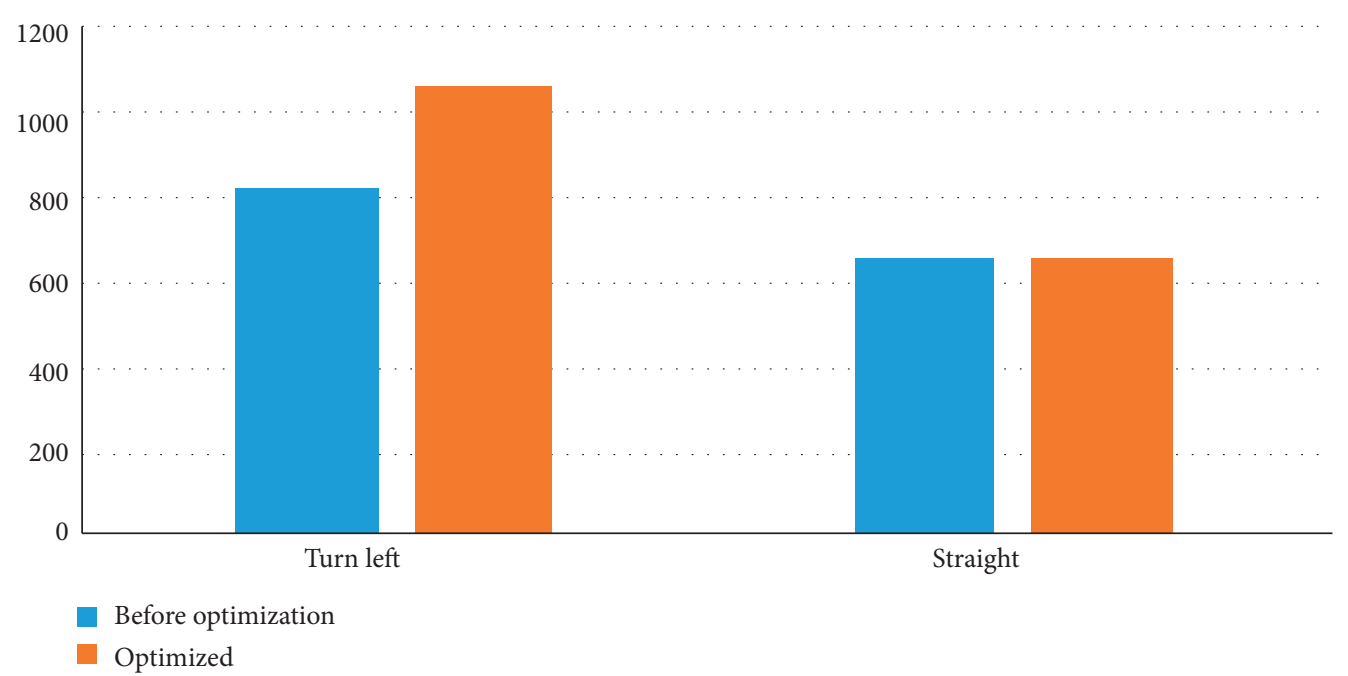

FIgURE 11: Comparison of saturation of each phase of Tangnan Road and Yongle East Road before and after optimization.

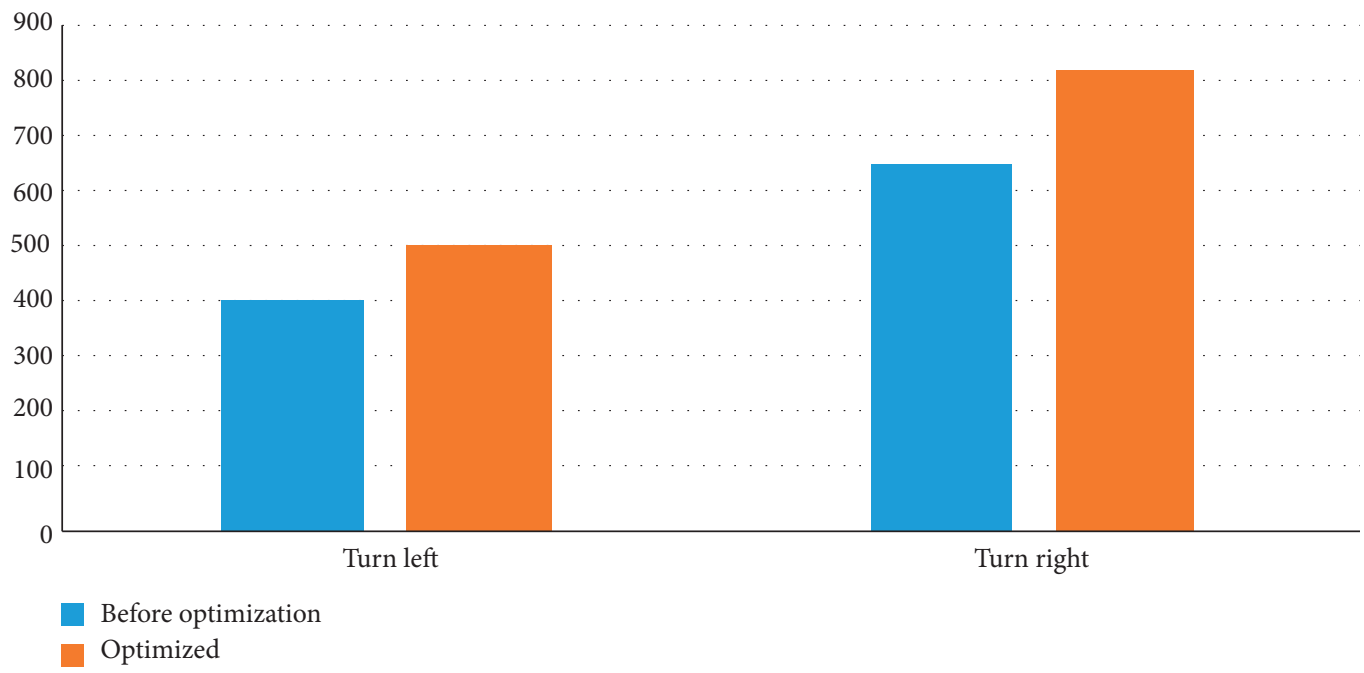

Figure 12: Comparison of saturation flow of each phase of Xingyuan Middle Road and Yongle East Road before and after optimization.

TABLE 8: Saturation flow rate after intersection optimization.

\begin{tabular}{lccc}
\hline Intersection parameter & Turn left & Straight & Turn right \\
\hline Intersection of Tongyang Road and Yongle East Road & 366 & 652 & - \\
Intersection of Tangnan Road and Yongle East Road & 1068 & 651 & - \\
Intersection of Xingyuan Middle Road and Yongle East Road & 515 & - & 817 \\
\hline
\end{tabular}

9.5\%. From Figure 8, we can see that the green wave bandwidth is $36 \mathrm{~s}$ and the green wave velocity is $37.9 \mathrm{~km} / \mathrm{h}$, and the free flow speed in Yongle East Road is $36 \mathrm{~km} / \mathrm{h}$, which indicates that, by implementing the green wave control, the speed index could be optimized 5.3\%. Tables 6 and 7 and Figure 9 show that, after implementing the green wave control and optimizing signal timing parameters and phase sequence at the three intersections on the Yongle East Road artery, the traffic capacity at the entrance of the intersections has been greatly improved, but from the comparison of Tables 1 and 7, it can be seen that the capacity of some phases at the intersection has not been improved obviously; for example, the improvement of the traffic capacity of the straight-through and left-turn phase at the intersection of Tongyang Road and Yongle East Road is not obvious, and the traffic capacity of the straight-through and left-turn phase at the intersection of Tangnan Road and Yongle East Road has not obviously improved. It can be seen from Figures 10-12 and Table 8 that the left-turn phase saturation flow at the intersection of Tongyang Road and Yongle East Road and the intersection of Tangnan Road and Yongle East Road has significantly improved. The left-turn 
phase and right-turn phase saturation flow at the intersection of Xingyuan Middle Road and Yongle East Road has been significantly improved.

\section{Conclusion}

In this paper, the urban road intersection group was taken as the research object, and the IVICS was used to analyze the capacity of the intersection group under the real-time reversible lanes to determine the calculation model of the capacity after setting the real-time reversible lanes in the IVICS. Based on the real-time reversible lane, the signal phase sequence and signal timing of the intersection group were designed, and the green wave control scheme was designed for the mainline based on the phase difference model. It constructed a phase difference collaborative optimization model between intersections and discussed signal timing parameter design and signal phase sequence design in low and high saturation states. Through the case study of green wave scheme design of Yongle East Road, Wuxi City, Jiangsu Province, the effectiveness of green wave control based on the phase difference model was verified using the number of stops, green wave speed, capacity, and saturation flow as evaluation indicators. The results show that the number of stops was optimized by $9.5 \%$, vehicle speed was improved by $5.3 \%$, and intersection capacity and saturated traffic have been greatly improved.

\section{Data Availability}

The data used to support the findings of this study are included within the article.

\section{Conflicts of Interest}

The authors declare that they have no conflicts of interest.

\section{Authors' Contributions}

Lina Mao and Guiliang Zhou designed and wrote the paper; Yuke Dai, Yao Liu, and Pengsen Hu conducted the model and collected traffic data; Yuke Dai and Xu Bao analyzed the simulation results.

\section{Acknowledgments}

This research was supported by the open fund for Jiangsu Key Laboratory of Traffic and Transportation Security (Huaiyin Institute of Technology) (TTS2020-05 and TTS2020-09), Enterprise-University-Research Institute Collaboration Project of Jiangsu Province (BY2020005), Graduate Innovative Projects of Jiangsu Province (KYLX15_0148), the National Natural Science Foundation of China (61573098 and 51308246), University Natural Science Major Basic Project of Jiangsu Province (15KJA580001), and the Natural Science Foundation of Jiangsu Province, China (BK20171426).

\section{References}

[1] M. W. Levin and S. D. Boyles, "A cell transmission model for dynamic lane reversal with autonomous vehicles," Transportation Research Part C, vol. 68, pp. 126-143, 2016.

[2] K. J. Assi and N. T. Ratrout, "Proposed quick method for applying dynamic lane assignment at signalized intersections," IATSS Research, vol. 42, no. 1, p. 7, 2018.

[3] W. K. M. Alhajyaseen, M. Najjar, and N. T. Ratrout, "The effectiveness of applying dynamic lane assignment at all approaches of signalized intersection," Case Studies on Transport Policy, vol. 5, no. 2, pp. 1-22, 2017.

[4] S. S. Dey, J. ma, and Y. Haden, "Reversible lane operation for arterial roadways: the Washington, DC, USA Experience," ITE Journal, vol. 81, no. 5, pp. 26-35, 2011.

[5] X. Cheng, H. Zhang, and W. Yu, "The construction and application of the phase difference model considering the influence of inbound and outbound traffic flow on the main line coordination," Science Technology and Engineering, vol. 19, no. 6, pp. 243-249, 2019.

[6] D. Qu, M. Wan, J. Li, J. Wang, and X. Xu, "Trunk line phase difference optimization and its control method based on traffic wave theory," Journal of Jilin University (Engineering and Technology Edition), vol. 47, no. 2, pp. 429-437, 2017.

[7] D. Qu, J. Yang, B. Qichun, W. Wang, and J. Zhou, "Phase difference optimization model based on queuing characteristics of trunk line traffic," Journal of Jilin University (Engineering and Technology Edition), vol. 48, no. 6, pp. 1685-1693, 2018.

[8] J. Cao, Cooperative Optimization Control Method for Lane Change Based on Tidal Flow Characteristics, Qingdao Technological University, Qingdao, China, 2016.

[9] F. Jiao, Reverse Variable Lane Dynamic Control Method Based on Multi-Source Data, Shandong University of Technology, Zibo, China, 2018.

[10] Y. Liu, Y. Chang, and S. Mao, "Research on signal timing optimization of intersections with reverse variable lanes," Journal of Chongqing University of Technology (Natural Science), vol. 32, no. 10, pp. 40-46, 2018.

[11] W. Liu, Z. Xie, and K. Chen, "Reverse variable lane signal timing optimization based on NSGA-II algorithm," Journal of Chongqing Jiaotong University (Natural Science Edition), vol. 37, no. 6, pp. 92-97, 2018.

[12] T. Jiang, Research on Variable Lane Setting Method of SignalControlled Level Crossing, Chang'an University, Xi'an, China, 2019.

[13] X. Zeng, J. Xu, and Z. Wu, "Optimal allocation of time and space resources at intersections based on variable lanes," Traffic Information and Safety, vol. 36, no. 06, pp. 81-89, 2018.

[14] H. Xu, Research on the Pre-signal Relationship of Variable Lanes at Signalized Intersections, Beijing University of Technology, Beijing, China, 2014.

[15] X. Li, J. Li, and M. He, "A coordinated control method of twoway green waves for trunk lines based on phase optimization," Journal of Traffic and Transportation Engineering and Information, vol. 16, no. 1, pp. 115-121, 2018.

[16] W. Wang, Traffic Engineering, Southeast University Press, Nanjing, China, 2011. 